\title{
Laser Treatment of Oral and Maxillofacial Hemangioma
}

\author{
Ehsan Azma ${ }^{1}$, Melika Razaghi ${ }^{*}$ \\ ${ }^{1}$ Oral and Maxillofacial Medicine of Dental Faculty of Guilan Medical University, Guilan, Iran \\ ${ }^{2}$ Laser Application in Medical Sciences Research Center, Shahid Beheshti University of Medical Sciences, Tehran, Iran
}

\author{
*Correspondence to \\ Melika Razaghi, DDS; Laser \\ Application in Medical Sciences \\ Research Center, Shahid Beheshti \\ University of Medical Sciences, \\ Tehran, Iran. \\ Tel: +98-9203629414; \\ Fax: +98-2122749221; \\ Email: razaghi.melika@ymail.com
}

Published online 17 September 2018

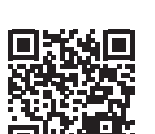

\begin{abstract}
Hemangioma is a congenital vascular soft tissue tumor, defined as a vascular lesion present in the newborn, with a progressive developmental pattern related to age. It has a progressive and regressive periodic growth mode, in comparison with arteriovenous malformation (AVM). Although there are many treatment approaches for curing this lesion such as; surgery, cryotherapy, sclerosant agents, laser therapy has more advantages in comparison to the other methods. Such as, hemostasis and clean operating field, decreased amount of pain and edema. There are many types of lasers manufactured that could be used for therapeutic purposes. This article focuses on different types of laser applications in the treatment of these lesions.
\end{abstract}

Keywords: Hemangioma; Vascular malformations; Laser therapy.

\section{Introduction}

Hemangioma is a congenital benign tumor of vascular tissue that arises from rapid endothelial cell proliferation followed by gradual involution. It can sometimes be missed at birth but then noticed during the first 8 weeks of life. There may be a regression period during adulthood. The progressive and developmental period occurs as age increases. ${ }^{1}$ These vascular anomalies have stricken up to $10 \%$ of newborns. The new classification of vascular anomalies identifies two main categories; vascular tumors and arteriovenous malformations (AVM). Hemangioma is a tumor of rapidly growing endothelial cells in which blood architectures have incomplete and hyperplasic cells, but vascular malformations do not contain hyperplasic cells and are formed by progressively enlarging aberrant and ectatic vessels composed of the type of vessel involved i.e. capillary, veins, arteriovenous.

Thus, these lesions are considered as their predominant vessel type i.e. venous malformations, AVMs or classified according to the hemodynamic features (low flow or high flow). ${ }^{2-4}$ Hemangioma is predominantly distributed on head and neck. ${ }^{5}$ The classic treatments for these lesions are sclerotherapy with injection of sclerosant agents and surgery that occasionally results in complications such as: significant deformity, prolonged pain, skin necrosis, nerve damage, systemic toxicity and hemorrhagic phenomenon. Because of these complications, laser therapy has been recently introduced as one of the main treatments of vascular lesions. ${ }^{6,7}$
Different kinds of laser systems have been applied for clinical use in treatment of hemangioma and vascular malformations since 1980s, such as carbon dioxide laser $\left(\mathrm{CO}_{2}\right)$, argon, diode, erbium-doped yttrium aluminum garnet (Er:YAG), potassium-titanyl-phosphate (KTP), pulsed dye laser (PDL) and neodymium-doped yttrium aluminium garnet (ND:YAG) lasers. ${ }^{8}$

\section{Laser Mechanism of Action}

Human maxillofacial tissues consist of different tissues; thus the clinician must first choose the appropriate laser for each treatment. Different types of lasers are applied in regard to specific tissues, e.g. for soft tissue treatments, the practitioner can use any available wavelength. All laser photons are absorbed by one or more of the soft tissue components. Lasers with short wavelengths (diode, Nd:YAG) are nonreactive with healthy tooth enamel; however, Er:YAG can remove hard and soft tissues near the operation site. Each laser's effect on tissue can be different, for example erbium and $\mathrm{Co}_{2}$ lasers are absorbed in high water contained tissues and because of their wavelengths, these lasers can penetrate only a few micron deep into the target tissue. Whereas the Nd:YAG laser can reach a few millimeters deep in the tissue. It should be considered that the relative penetration of different wavelengths varies. Also the penetration of laser photons into mucosa and tissue may occur beyond the surgical field. ${ }^{9}$

The final aim of laser therapy for hemangioma and 
other similar lesions is the selective destruction of tumoral vessels through the absorption of laser photons by hemoglobin molecules in red blood cells. Thermal energy of laser photons scatters radially within the blood vessel inducing selective microvascular hazardous effects, through photocoagulation and mechanical injury. The chosen wavelength should be absorbed selectively by hemoglobin molecules. Several factors such as the size of vessels, depth of the lesion, area of body treated, laser spot size, skin type and fluence can affect the absorption of laser. There are several types of lasers used in laser surgery. There are 3 types of mode of operation including continuous wave lasers (CWLs) such as $\mathrm{CO}_{2}$, argon and diode laser, pulsed laser systems such as Nd:YAG and Er:YAG lasers, chopped mode which is a variety of continuous mode that have equal power photons, but have many equal distance interruptions in milliseconds. ${ }^{10-12}$

\section{Argon Laser}

This type of laser has a wavelength of $514 \mathrm{~nm}$ and is observed as green light. This laser is able to penetrate into the skin and mucosa to a depth of $0.5 \mathrm{~mm}$ and photocoagulate the hemoglobin molecules in the vessels. Thus, this laser can microscopically remove superficial hemangioma. The laser photons are absorbed by hemoglobin and cause coagulation necrosis of dermis, and vascular embolization of the papillary layer without harm to cutaneous appendages. This type of laser has a precise effect for removing superficial telangiectasia and small, flat hemangioma, but in deeper and larger hemangioma it has a variable response, for which it has recently been replaced with pulsed lasers. ${ }^{5,10}$

\section{Flashlamp-Pumped Pulse Dye Laser}

This laser was designed for cutaneous vascular lesions treatment without scar formation. This type of laser has 2 wavelengths of 585 and $595 \mathrm{~nm}$. The laser photons destruct blood vessels by using an exposure time less than the calculated thermal relaxation time $(1-10 \mathrm{~ms})$ for the blood vessels. It was advocated that an exposure time of a millisecond or less is required to confine the heat to the vessel in order to decrease the risk of heat diffusion, and skin scarring. This laser can induce thrombosis within the vessels without damage to the dermis. With use of $585 \mathrm{~nm}$, the penetration depth for $50 \%$ of the energy is calculated to be $0.8 \mathrm{~mm}$. On the other hand, the dermis of facial skin is approximately $0.6 \mathrm{~mm}$ deep in children and $0.9 \mathrm{~mm}$ in adults. Therefore, FPDL has proved to be a safe and effective treatment modality for superficial hemangioma in the face. Immediately after laser radiation, the color of the treated area turns to blue-lilac (purpuric) color, with surrounding erythematous flare which resolves in 7 to 14 days after laser therapy. The side effects comprise edema, especially in the periorbital area. If the epidermis becomes blanching or graying during application, the energy fluences should be reduced to avoid blisters. After treatment, the treated areas are covered with panthenol ointment in order to improve hydration, reduce itching and inflammation of the skin, and accelerate epidermal wound healing. In a condition were blistering or crusting occurs, povidone-iodine solution is recommended. The application of this type of laser has no benefit in deeper procedures. The superficial compartment resolve but the deeper compartment continues to proliferate or not be affected during laser therapy. Therefore, it has been suggested to use corticosteroids and Nd:YAG laser for treatment of these types or sequential use of PDL with wavelength of $595 \mathrm{~nm}$ and Nd:YAG laser in treatment of lesions resistant to conventional treatment of PDL. ${ }^{13-15}$

\section{Nd:YAG Laser}

Nd:YAG laser is a kind of solid state laser that has a wavelength of $1064 \mathrm{~nm}$ which emits an infrared invisible light in continuous wave with a penetration depth of $1.0 \mathrm{~mm}$. The laser energy can be absorbed mostly by blood vessels, the laser photons are also absorbed in surrounding tissues. This type of laser can penetrate in deeper lesions and create photocoagulation and hemostasis with little edema and bleeding because of its wavelength. Ice cubes can be used to cool the area during irradiation for prevention of damage to surrounding tissues. The percutaneous or intralesional application technique is a valuable tool for subcutaneous or mixed hemangioma cases with larger or deeper compartments ( $2 \mathrm{~cm}$ in depth). The laser beam has a spot diameter with a size of $2 \mathrm{~mm}$ on the surface of the lesion. The output power of the laser is variable between 20 to $50 \mathrm{~W}$. For the treatment of cases with smaller deep red hemangiomas, the lower range of output power i.e. 20 to $34 \mathrm{~W}$ is chosen with continuous cooling using ice cubes. However, in thicker subcutaneous hemangiomas, a higher output power about $50 \mathrm{~W}$ is applied with continuous ice cooling. Subcutaneous hemangiomas should be treated in repeated sessions with interval treatment course of 4 to 6 weeks. Intraoral hemangioma has been treated with output power of 4 to $8 \mathrm{~W}$ in many sessions and short times without cooling. Unfortunately, some of these lesions almost develop rapidly, and repeated laser treatment is helpful to slow progress of this process and to accelerate resolution. ${ }^{16}$ Treatment of intraoral lesions occurs through vaporization or excision depending on the size and proximity of the lesions to vital compartment e.g. neural, vascular, and salivary. ${ }^{17}$ The treatment is done in phases every three months to control and examine the reduction of growth and size of the lesion in the buccal, lingual, gingival, and palatal areas.

The settings with optimum results are an output power of $400-600 \mathrm{~J} / \mathrm{cm}^{2}$ with pulse duration of 0.5 seconds in surface noncontact application mode., ${ }^{2,18}$ This treatment is very painful and should be performed under general anesthesia. After treatment, the lesion area becomes swollen and will acquire a rigid consistency immediately. 
The blistering or a scab on the surface of affected area may appear. The swelling often resolves within 1 week after treatment, and the lesion becomes flat and blanched during 2 to 4 weeks post-treatment. The response rate of laser therapy for hemangioma is variable between $77 \%$ and $100 \%$. The size of hemangioma at initial treatment is an important issue, the smaller size lesions have better therapeutic results. ${ }^{19}$ There are some complications that have been reported such as; hyper-pigmentation, scarring, atrophy of operating tissue, and dyspigmentation which is due to spontaneous remission of large hemangioma. ${ }^{5,18}$

\section{$\mathrm{CO}_{2}$ Laser}

This laser has a wavelength of $10600 \mathrm{~nm}$ and the best absorption in high water composed tissues and is considered as an ideal laser in soft tissue surgery. This type of laser has two modes; continuous wave and ultra-pulsed system. In continuous mode, production of equal beams of light causes rapid tissue vaporization and cellular destruction occurs through photothermal effect. The cells absorb photons' energy, which leads to vaporization of cellular water and cellular interruption before conduction of heat in surrounding tissue, creating a zone of necrosis about $500 \mu \mathrm{m}$ or less. The composition of pigmentation and vascularization is not important in $\mathrm{Co}_{2}$ laser application. The coagulation of vessels up to $500 \mu \mathrm{m}$ diameters and hemostasis reduces postsurgical bacteremia better than other surgical methods.

This laser improves the surface texture appearance. ${ }^{10,20}$ This type of laser is successfully used for tongue reduction in macroglossia, and also in treatment of intraoral hemangioma, especially tongue and lips. The excision of tumor, hemostasis by coagulation and vaporization, in addition to the safety, easily performed operation and diminished hospitalization and medical expenses for the patient makes this type of laser one of the best methods for treatment of hemangioma. While $\mathrm{Co}_{2}$ laser is more used in treatment of superficial hemangioma, the healing period is longer than in other methods. ${ }^{21,22}$

\section{KTP Laser}

This laser has double the frequency of Nd:YAG laser and is one of the solid state lasers. It has a wavelength of $532 \mathrm{~nm}$ and emits short pulses and high peak power photons that differ from argon laser and is observed as green light. The clinical effect has been noted to be between argon laser and flashed-pumped PDL. ${ }^{23}$ This wavelength is in about the hemoglobin absorption peak and high absorption of photons by hemoglobin occurs. This laser is a suitable vehicle for treatment of superficial hemangioma. It has a wide range of pulse duration from 1 to $100 \mathrm{~ms}$ which leads to longer pulse duration and slow heat conduction to blood vessel without rupture of blood vessel walls. Because of deeper penetration and coagulation, it is better than $\mathrm{Co}_{2}$ laser application, especially in subglottic area. However this laser has many complications such as edema, purpura and crusting. ${ }^{16,24}$

\section{Er:YAG Laser}

This laser type belongs to solid state lasers and the erbium family laser which has two wavelengths, the Er:YAG with $2940 \mathrm{~nm}$ wavelength, and the Er-Cr:YAG with $2780 \mathrm{~nm}$ wavelength. Both of them have pulsed mode of operation and delivery systems as mirrored hand-pieces with articulated arm or fibers conduction systems. These lasers have highest absorption in water and hydroxyapatite ions. They have been applied for removing hard and soft tissues of oral cavity such as dental caries, osteoplasty, exophytic tissue and frenectomy. Vaporization of water molecules within hard tissues creates micro-explosions in the hydroxy-apatite molecules (ablation phenomenon) leading to a break down of hard tissue without charring and carbonization, while causing minimal heat generation. The erbium lasers ablate soft tissue and are most effective in lightly vascularized tissue where bleeding will not be an issue and has been least effective in achieving hemostasis, but Er-Cr: YSGG is more efficient than Er:YAG and is used in gingivectomy, frenectomy and crown lengthening. The delivery systems comprise a water spray to prevent heat damage to tissues, rehydrating target tissues in order for photon's energy to be better absorbed by the target organ. Interstitial photocoagulation is produced in treatment of hemangioma like with other lasers such as; ND:YAG laser which led to regression of lesion and no reperfusion, also no scar formation has been reported..$^{10,15}$

\section{Intense Pulsed Light}

This laser has high-intensity light sources that can emit polychromatic light. Unlike other laser systems, these flash lamps exit non-coherent light in wavelength spectrum from $500 \mathrm{~nm}$ to over $1100 \mathrm{~nm}$ and working energies up to $80 \mathrm{~J} / \mathrm{cm}^{2}$. There are many filters that minimize the emitted light in range of 515 and $590 \mathrm{~nm}$. The emitted light may be single, double, or triple pulses in the millisecond range. These pulse durations protect the outer epidermis. These adjustable wavelengths and pulse durations provide good diversity in the treatment of a variety of lesions such as vascular and pigmented lesions for different skin types. This system has a cooling device, but generally the temporary erythema is the only acute side effect. On the basis of properties of this laser, treatment of benign cavernous hemangioma, benign venous malformations, essential facial telangiectasia and port wine stains are possible. ${ }^{26}$

\section{Laser Treatment of Port-Wine Stains}

Port-wine stain (PWS) is defined as birthmarks or vascular malformations consisting of ectatic blood vessels in the papillary and upper reticular dermis. Many types of lasers were applied for treatment of this disorder such as $\mathrm{CO}_{2}$, $\mathrm{Nd}$ :YAG and cooper vapor laser, but the PDL therapy is the standard approach for treating these birthmarks. The success rate of total treatment with the PWS is about $10 \%$. The result of treatment is different in individuals and sites from person to person. The presumed aim of 
laser treatment is acute photocoagulation of the PWS microvasculature. Clinically a common sign used to assess photocoagulation is purpura (bluish-gray skin discoloration from blood extravasation) formation, although the purpura formation may occur without photocoagulation. ${ }^{25}$ The incidence of removing lesion by this technique is as yet un-documented. On the other hand, red lesions are more resistant to treatment than the light pink lesions. Recently, it has been recognized that the degree of response correlates to the anatomical location of the PWS. Also some researches revealed that patients under 4 years of age need less treatment to reach the same degree of response. The mid-face location of port wine stain has slower response to pulse dye laser treatment compared to other locations. ${ }^{4,27}$

During laser treatment, the cooling chamber leads to significant reduction of pain sensation. The infants over 7 years can often be treated without anesthesia, although this is related to the size and anatomic site of the lesion. While in child cases with extensive lesions or in central facial PWSs, general anesthesia especially for eye protection is beneficial. The treatment of port wine stain should take place as early as possible, since in addition to the abnormality of face, the port wine stain has a considerable degree of psychological morbidity. ${ }^{27}$

\section{Comparisons of Lasers}

Hemangioma treatment with laser systems has different outcomes with one laser system having better therapeutic effect in the treatment course. PDL laser system has the best documented safety record in laser systems. Another advantage of this laser is its application for different age groups. Laser therapeutic effects are almost limited to the superficial portions of hemangioma. Laser treatments with PDL system do not diminish the development of deeper content of hemangioma. It is difficult to deal with deeper components in hemangioma, so the deeper component of the lesion may still grow after treatment, in spite of the superficial component having been successfully treated. The effectiveness of PDL on deeper component of hemangioma is not well established, lesions thicker than $3 \mathrm{~mm}$ may not respond well to PDL. Superficial ectatic blood vessels of incompletely regressed hemangioma can be treated with PDL. In the laser treatment of childhood hemangioma, it was seen that the application of long pulsed PDL is safer and more effective than PDL. For deep components of hemangioma, the longer-wavelength, deeper-penetrating lasers, such as the Nd:YAG laser, have been tried with variable results. Ulcerated hemangiomas are painful and require local anesthesia (1\% lignocaine). Lesions involving larger areas need topical, local or general anesthesia

It has been proven that Nd:YAG laser therapy can be effective in the treatment of superficial venous malformations, but in deeper lesions because of photon energy absorption in skin and mucosa, low level laser therapy in many sessions may be beneficial in the shrinkage of hemangioma. ${ }^{19}$ In a research conducted by Raulin and $\mathrm{Greve}^{28}$ the comparison of the efficacy of PDL with long-pulsed frequency-doubled $\mathrm{Nd}$ :YAG were evaluated, the result of this research showed the regression rate of hemangioma was somewhat higher with PDL laser usage. In Poetke and Berlien study, ${ }^{39}$ the excellent response in treatment of hemangioma was 53\%. In another studides the application of PDL was quick, effective, and almost devoided of side effects and specially showed improved effects in treatment of ulcerated hemangioma. ${ }^{30-33} \mathrm{Nd}$ :YAG has many side effects such as swelling, blistering, crust formation, which was more than PDL laser, on the other hand purpura formation, hypo and hyperpigmentation creation were more than Nd:YAG laser. These side effects in Nd:YAG laser applications are due to deep thermal hazards from intensely penetrating infrared light wavelength of this laser type. Nd:YAG laser has a narrow band of safety and efficacy. Researchers insist on the KTP laser as an efficient treatment of deep hemangiomas, because this laser system can be used with the low output power 2 to $5 \mathrm{~W}$ ) and the ulceration rate is decreased from $20 \%$ to $2 \%$.

The smaller output power leads to selective absorption rather than thermal effect and low ulceration rate, as well as, both the superficial and deep components of the hemangioma can be treated together simultaneously. ${ }^{30}$

\section{Contraindication of laser therapy in hemangioma treatment}

There are some contraindications that are both absolute and relative, the absolute contraindications are: Active local infection or photo-aggravated skin diseases and medical conditions. The relative contraindications are: unstable vitiligo, psoriasis, keloid and keloidal tendencies, patient on isotretinoin or patient who is not cooperative or has unrealistic expectations. ${ }^{31}$

\section{Conclusion}

There are many laser systems used in the treatment of hemangioma and vascular tumors. These laser systems have different wavelengths and penetration depths, however, they have similar mechanisms and in some cases two or more lasers can be applied during the course of treating these lesions.

\section{Ethical Issues}

Not applicable.

\section{Conflict of interests}

None.

\section{References}

1. Neville BW, Damm DD, Allen CM, Bouquot JE. Oral and Maxillofacial pathology. 3rd ed. St Louis, Missouri: Saunders Elsevier; 2009:538-547. 
2. Buckmiller LM, Richter GT, Suen JY. Diagnosis and management of hemangiomas and vascular malformations of the head and neck. Oral Dis. 2010;16(5):405-418. doi:10.1111/j.1601-0825.2010.01661.x.

3. Greene AK, Kim S, Rogers GF, Fishman SJ, Olsen BR, Mulliken JB. Risk of vascular anomalies with Down syndrome. Pediatrics. 2008;121(1):e135-140. doi:10.1542/ peds.2007-1316.

4. Kohout MP, Hansen M, Pribaz JJ, Mulliken JB. Arteriovenous malformations of the head and neck: natural history and management. Plast Reconstr Surg. 1998;102(3):643-654.

5. Zheng JW, Zhou GY, Wang YA, Zhang ZY. Management of head and neck hemangiomas in China. Chin Med J (Engl). 2008;121(11):1037-1042.

6. Lapidoth M, Yaniv E, Ben Amitai D, et al. Treatment of facial venous malformations with combined radiofrequency current and $900 \mathrm{~nm}$ diode laser. Dermatol Surg. 2005;31(10):1308-1312.

7. Scherer K, Waner M. Nd:YAG lasers $(1,064 \mathrm{~nm})$ in the treatment of venous malformations of the face and neck: challenges and benefits. Lasers Med Sci. 2007;22(2):119126. doi:10.1007/s10103-007-0443-0.

8. Crisan BV, Baciut M, Baciut G, Campian RS, Crisan L. Laser treatment in oral and maxillofacial hemangioma and vascular malformations. TMJ. 2010; 60(1):34-38.

9. Coluzzi DJ. Fundamentals of lasers in dentistry: basic science, tissue interaction, and instrumentation. J Laser Dent. 2008;16:4-10.

10. Convissar RA. The Principles and Practice of Laser Dentistry. 1st ed. Mosby elsevier St Louis Missouri. 2011:248.

11. Niamtu J 3rd. The treatment of vascular and pigmented lesions in oral and maxillofacial surgery. Oral Maxillofac Surg Clin North Am. 2004;16(2):239-254. doi:10.1016/j. coms.2004.01.006.

12. Astner S, Anderson RR. Treating vascular lesions. Dermatol Ther. 2005;18(3):267-281. doi:10.1111/j.15298019.2005.05025.x.

13. Anderson RR, Parrish JA. The optics of human skin. $J$ Invest Dermatol. 1981;77(1):13-19.

14. Boixeda P, Carmona LP, Vano-Galvan S, Jaén P, Lanigan SW. Advances in treatment of cutaneous and subcutaneous vascular anomalies by pulsed dual wavelength 595- and 1064-nm application. Med Laser Appl. 2008;23(3):121-126. doi:10.1016/j.mla.2008.04.002.

15. Railan D, Parlette EC, Uebelhoer NS, Rohrer TE. Laser treatment of vascular lesions. Clin Dermatol. 2006;24(1):815. doi:10.1016/j.clindermatol.2005.10.026.

16. Vesnaver A, Dovsak DA. Treatment of vascular lesions in the head and neck using Nd:YAG laser. J Craniomaxillofac Surg. 2006;34(1):17-24. doi:10.1016/j.jcms.2005.07.009.

17. Wlodawsky RN, Strauss RA. Intraoral laser surgery. Oral Maxillofac Surg Clin North Am. 2004;16(2):149-163. doi:10.1016/j.coms.2004.02.001.

18. Iannetti G, Torroni A, Chiummariello S, Cavallotti C. Clinical and morphological characteristics of headfacial haemangiomas. Head Face Med. 2007;3:12. doi:10.1186/1746-160x-3-12.

19. Zheng JW, Zhou Q, Yang XJ, et al. Treatment guideline for hemangiomas and vascular malformations of the head and neck. Head Neck. 2010;32(8):1088-1098. doi:10.1002/ hed.21274.

20. Brauer JA, Geronemus RG. Laser treatment in the management of infantile hemangiomas and capillary vascular malformations. Tech Vasc Interv Radiol. 2013;16(1):51-54. doi:10.1053/j.tvir.2013.01.007.

21. Lambrecht JT, Stubinger S, Hodel Y. [Treatment of intraoral hemangiomas with the CO2 laser]. Schweiz Monatsschr Zahnmed. 2004;114(4):348-359.

22. Apfelberg DB, Maser MR, Lash H, White DN. Benefits of the CO2 laser in oral hemangioma excision. Plast Reconstr Surg. 1985;75(1):46-50.

23. Poetke M, Berlien HP. Laser treatment in hemangiomas and vascular malformations. Med Laser Appl. 2005;20(2):95102. doi:10.1016/j.mla.2005.03.008.

24. Kacker A, April M, Ward RF. Use of potassium titanyl phosphate (KTP) laser in management of subglottic hemangiomas. Int $J$ Pediatr Otorhinolaryngol. 2001;59(1):15-21.

25. Huang YC, Tran N, Shumaker PR, et al. Blood flow dynamics after laser therapy of port wine stain birthmarks. Lasers Surg Med. 2009;41(8):563-571. doi:10.1002/ $1 \mathrm{sm} .20840$.

26. Goldberg DJ. Laser removal of pigmented and vascular lesions. J Cosmet Dermatol. 2006;5(3):204-209. doi:10.1111/ j.1473-2165.2006.00252.x.

27. Renfro L, Geronemus RG. Anatomical differences of portwine stains in response to treatment with the pulsed dye laser. Arch Dermatol. 1993;129(2):182-188.

28. Raulin C, Greve B. Retrospective clinical comparison of hemangioma treatment by flashlamp-pumped (585 nm) and frequency-doubled Nd:YAG $(532 \mathrm{~nm})$ lasers. Lasers Surg Med. 2001;28(1):40-43.

29. Poetke $M$, Berlien HP. Treatment of a subungual hemangioma with flashlamp-pumped pulsed-dye laser. $J$ Am Acad Dermatol. 2000;43(6):1135-1136.

30. Stier MF, Glick SA, Hirsch RJ. Laser treatment of pediatric vascular lesions: Port wine stains and hemangiomas. $J$ Am Acad Dermatol. 2008;58(2):261-285. doi:10.1016/j. jaad.2007.10.492.

31. Srinivas CR, Kumaresan M. Lasers for vascular lesions: standard guidelines of care. Indian J Dermatol Venereol Leprol. 2011;77(3):349-368. doi:10.4103/0378-6323.79728.

32. Hohenleutner S, Badur-Ganter E, Landthaler $M$, Hohenleutner U. Long-term results in the treatment of childhood hemangioma with the flashlamp-pumped pulsed dye laser: an evaluation of 617 cases. Lasers Surg Med. 2001;28(3):273-277. doi:10.1002/lsm.1050.

33. Batta K, Goodyear HM, Moss C, Williams HC, Hiller L, Waters R. Randomised controlled study of early pulsed dye laser treatment of uncomplicated childhood haemangiomas: results of a 1-year analysis. Lancet. 2002;360(9332):521527. doi:10.1016/s0140-6736(02)09741-6. 